\title{
ATRIBUIÇÕES DO ENFERMEIRO EM UM PROGRAMA DE ATENÇÃO DOMICILIAR DO SISTEMA ÚNICO DE SAÚDE
}

\author{
NURSES' ASSIGNMENTS IN A HOME CARE PROGRAM \\ OF THE BRAZILIAN'S PUBLIC HEALTH SYSTEM
}

\section{Denis Fernandes da Silva Ribeiro ${ }^{a}$, Germana Périssé de Abreu ${ }^{b}$}

adenisfernandesr@hotmail.com, bribeirodfs.enf@gmail.com

Universidade do Grande Rio - Duque de Caxias (RJ), Brasil

Data de recebimento do artigo: 02/05/2016

Data de aceite do artigo: 22/02/2017

\section{RESUMO}

Este trabalho objetiva descrever o funcionamento de um programa de atenção domiciliar vinculado ao Sistema Único de Saúde (SUS) e apontar as atribuiçóes desempenhadas pelo enfermeiro dentro desse programa. Trata-se de um estudo exploratório e descritivo. O cenário de coleta de dados foi um programa de atençấo domiciliar (PAD) do município do Rio de janeiro (RJ), no qual se procedeu levantamento documental e acompanhamento de visitas domiciliares randômicas, utilizando-se da observação sistemática não participante para levantar as atribuiçốes do enfermeiro. O projeto foi aprovado pelo Comitê de Ética em Pesquisa em Seres Humanos da Secretaria Municipal de Saúde do Rio de Janeiro, sob o parecer $\mathrm{n}^{\circ}$ 775.267. A atenção domiciliar no Rio de Janeiro é priorizada aos idosos e funciona desde o ano de 2010 sob o Programa de Atenção Domiciliar do Idoso, com cinco bases distribuídas de forma equânime no município. Nesse cenário, o enfermeiro ocupa cargos assistenciais e de gestáo, desenvolvendo a assistência, açóes com ênfase ao paciente, a família e o domicílio. Conclui-se que compreender as atribuiçôes do enfermeiro nesse programa contribui para delimitar a atuação profissional com perícia, além de subsidiar a formação e o aperfeiçoamento profissional.

Palavras-chave: Serviços de assistência domiciliar; cuidados de enfermagem; serviços de saúde para idosos.

\section{ABSTRACT}

This article aims to describe the operation of a home care program linked to the Brazilian's Unified Health System and to point the assignments performed by nurses in it. It is an exploratory and descriptive study. The research's scenario was a Home Care Program of the municipality of Rio de Janeiro (RJ), and the collected data include a documentary survey and the monitoring of random home visits, in which we used the systematic non-participant observation to survey the nurses' assignments. The project was approved under permit $\mathrm{n}^{\circ} \mathbf{7 7 5 . 2 6 7}$, by the Human Research Ethics Committee of Municipal Health Secretariat of Rio de Janeiro. The home care in Rio de Janeiro is a priority to the elderly and has been operating since 2010, under the Programa de Atenção Domiciliar do Idoso (Portuguese for "Home Care Program for the Elderly"), with five bases distributed equitably in the city. In this scenario, the nurse takes care and management positions, developing in care actions, with emphasis on the patient, family, and household. We conclude that understanding the duties of nurses in this program contributes to define their professional practice with expertize, support training, and professional development.

Keywords: Home care services; nursing care; health services for eldery people. 


\section{Introdução}

O envelhecimento populacional já se mostra uma realidade em todo o Brasil, sendo o produto da queda nas taxas de fecundidade e mortalidade, além dos progressos sanitários que ocorreram nas últimas décadas ${ }^{1}$. Em alguns municípios do Brasil, a população é nitidamente envelhecida, e este é o caso do Rio de Janeiro, que detém importante concentraçáo de idosos, correspondendo, de acordo com o Censo Demográfico de 2010 do Instituto Brasileiro de Geografia e Estatística (IBGE), a $14,9 \%$ da população ${ }^{2}$.

O envelhecer é um processo fisiológico e, assim como as demais etapas da vida, implica suas próprias características e demandas biopsicossociais, requerendo a reformulação de políticas públicas e a elaboração de diferentes estratégias de atençãa ${ }^{3}$. Essas são as principais demandas da populaçáo idosa relacionadas à assistência social e saúde, frente à dificuldade no acesso aos serviços básicos e a disparidade social que ocorre no Brasil ${ }^{4}$.

A atenção domiciliar é uma das estratégias implementadas pelo setor saúde, tendo em vista a transição demográfica e epidemiológica que decorre no Brasil, isto é, a mudança para uma populaçáo idosa e um maior acometimento de agravos crônicos não transmissíveis ${ }^{3}$. É uma modalidade de assistência à saúde em expansão no país e que vem ganhando visibilidade desde o ano de 2011, com a instituição do Programa Melhor em Casa, pelo Ministério da Saúde .

Para o Ministério da Saúde ${ }^{6}$, a atenção domiciliar é um conjunto de açôes de promoção à saúde, prevenção e tratamento de doenças e reabilitação proporcionadas em domicílio, de caráter substitutivo ou suplementar e continuamente integrado com as redes de atenção à saúde. Esse tipo de assistência é desenvolvido nos diferentes níveis de atenção à saúde e possibilita práticas mais próximas do conceito de integralidade ${ }^{7}$.

Dentro das redes de atenção à saúde do âmbito do SUS, a atenção domiciliar articula suas ações por meio dos serviços primários, dos dispositivos ambulatoriais, de urgências e emergências hospitalares, tendo a atenção primária à saúde como ordenadora do cuidado. As açóes e responsabilidades de cada ponto de atenção da rede são pactuadas através de fluxos e protocolos conjuntos, de acordo com as três modalidades de atenção domiciliar: $\mathrm{AD} 1, \mathrm{AD} 2$ e $\mathrm{AD} 3^{8}$.

As unidades primárias de saúde são responsáveis pela prestação do nível menos complexo de atenção domiciliar, que é a modalidade AD1. Já as modalidades AD2 e AD3, que são mais complexas, são disponíveis apenas pelos serviços de atenção domiciliar (SAD), que podem ser vinculados a hospitais, ambulatórios, e serviços de atenção às urgências e emergências ${ }^{6}$.
Nessa perspectiva, a atenção domiciliar permite diminuir os episódios de hospitalização, reagudizaçáo e (re)internaçóes, além de otimizar custos ao serviço de saúde e minimizar o risco de infecçóes nosocomiais. Favorece, ainda, uma assistência integral, humanizada e resolutiva, que por sua vez propicia satisfaçáo e maior qualidade de vida ao paciente e eixo familiar ${ }^{7,9,10}$.

Dessa maneira, a atenção domiciliar desponta como cenário inovador para a prática do cuidado, em que há novos modos de trabalho e práticas profissionais, focando os aspectos multidimensionais envolvidos no processo saúde-doença do sujeito e família. Esses aspectos são abordados por um processo interdisciplinar, no intuito de proporcionar integralidade da assistência, com estímulo à participaçáo, corresponsabilizaçáo, autonomia $\mathrm{e}$ independência ${ }^{7,11,12}$.

As equipes nos SAD dividem-se em equipes multiprofissionais de atenção domiciliar (Emad); e em equipes multiprofissionais de apoio (Emap). As Emad são compostas obrigatoriamente por: auxiliares/técnicos de enfermagem, enfermeiro, médico, podendo incluir fisioterapeuta ou assistente social. Já as Emap são constituídas minimamente por três dos seguintes profissionais: assistente social, fisioterapeuta, fonoaudiólogo, nutricionista, odontólogo, psicólogo, farmacêutico e terapeuta ocupacional ${ }^{6}$.

A interdisciplinaridade que é permitida na atenção domiciliar se mostra relevante para recuperar o estado de saúde aos usuários, uma vez que o indivíduo é assistido em sua totalidade, sendo cada ação, atribuição e habilidade profissional responsável para atingir tal resultado. Essa articulação dos diferentes saberes e práticas contribui para extrapolar as práticas biologicistas ${ }^{11}$.

Sabe-se que a enfermagem é a classe profissional que realiza o maior número de procedimentos e que mais despende tempo dentro da atençáo domiciliar ${ }^{12}$, inserindo-se nesse cenário de cuidado com diferentes graus de participação e possibilidades de atuação ${ }^{13}$, que englobam: consultas primárias e subsequentes; cuidado com curativos, ostomias, sondas, cateteres e drenos; administração e orientação sobre medicamentos prescritos; entre outros ${ }^{12}$.

Este estudo se justifica pelo interesse dos autores em pesquisar as atribuições desempenhadas pelo enfermeiro na atenção domiciliar, bem como o estabelecimento desta no município do Rio de Janeiro, tendo em vista a relevância atual dessa modalidade de saúde como cenário inovador de práticas de cuidado e a necessidade de exploração da temática.

Dessa maneira, este estudo objetiva descrever o funcionamento de um programa de atenção domiciliar (PAD) vinculado ao SUS, apontando as atribuiçóes desempenhadas por um enfermeiro integrante desse programa. 


\section{Materiais e métodos}

O presente estudo é exploratório e descritivo. O cenário de coleta de dados foi uma das bases de um PAD do SUS no município do Rio de Janeiro. A coleta de dados foi feita em duas etapas: levantamento documental e observação sistemática da prática do enfermeiro do referido serviço, ocorrendo entre os meses de setembro e dezembro do ano de 2015.

$\mathrm{Na}$ primeira etapa, a fase documental da pesquisa, procedeu-se a exploraçáo dos materiais e arquivos para levantar os instrumentos normativos e legislaçóes que instituíram e fundamentam o funcionamento do referido PAD no município do Rio de Janeiro. Também foi realizado um procedimento de revisão de literatura para o levantamento teórico do estado da arte da temática e também para a confecção o instrumento de observação sistemática da prática do enfermeiro nesse serviço.

Buscou-se nas bases de dados associadas à Biblioteca Virtual em Saúde sobre os descritores "serviços de assistência domiciliar" e "cuidados de enfermagem", tendo os seguintes critérios de inclusão: trabalhos publicados em português; disponíveis on-line e gratuitamente; e publicados entre os anos de 2010 e 2015. Critérios de exclusão: trabalhos que náo estivessem relacionados à saúde do idoso. Teve-se como resultado 18 trabalhos, sendo selecionados 9 após leitura prévia. Predominaram artigos publicados na Literatura Latino-Americana e do Caribe em Ciências da Saúde (Lilacs) e BDENF - enfermagem.

A técnica de observação empregada foi sistemática, pautada através de roteiro-guia e pelo método não participante. Os autores construíram um roteiro-guia de observação tendo como arcabouço teórico as publicaçóes do Ministério da Saúde sobre o Programa Melhor em Casa, bem como a partir das legislaçôes da atenção domiciliar. O referido roteiro abordou as açóes desempenhadas pelo enfermeiro nas visitas domiciliares e na modalidade da sistematização da assistência, bem como a interação profissional do enfermeiro com os demais profissionais.

A observação da prática do enfermeiro no PAD ocorreu com o acompanhamento de cinco visitas domiciliares randômicas, em dias intercalados por períodos irregulares, entre os meses de setembro e outubro de 2015. Os atendimentos domiciliares realizados pelo enfermeiro foram, então, observados de modo que foi possível identificar as atribuições do perfil e a relevância do cuidado de enfermagem prestado pelo enfermeiro nessa modalidade de atenção à saúde. Os resultados foram analisados qualitativamente à luz da literatura de enfermagem, das publicaçóes mais recentes do Ministério da Saúde sobre a atenção do domiciliar e da atenção à saúde do idoso.
O projeto de pesquisa atendeu aos princípios éticos requeridos pela Resolução do Conselho Nacional de Saúde de $n^{\circ} 466 / 12$, sendo submetido via plataforma Brasil ao Comitê de Ética em Pesquisa em Seres Humanos da Secretaria Municipal de Saúde do Rio de Janeiro e aprovado sob o CAAE no 34601514.2.0000.5279. A coleta de dados dos profissionais se iniciou exclusivamente após a assinatura do termo de consentimento livre e esclarecido.

\section{Resultados e discussão}

Os achados deste estudo serão apontados em duas partes: a primeira é produto do levantamento documental e é composta pelo panorama de funcionamento do Programa de Atenção Domiciliar instituído no município do Rio de Janeiro (RJ), suas consonâncias e discrepâncias com o proposto pelo Programa Melhor em Casa do Ministério da Saúde, bem como o desempenho desse serviço dentro das redes de atenção à saúde no município. A segunda é proveniente da observação sistemática, sendo composta das atribuiçóes desempenhadas pelo enfermeiro nesse programa de atenção domiciliar.

\section{Programa de Atenção Domiciliar ao Idoso}

O serviço de atenção domiciliar do município do Rio de Janeiro é denominado Programa de Atenção Domiciliar ao Idoso (Padi), com o intuito de apontar a faixa etária a que é prioritário e direcionado. Ressaltase que esse serviço não é exclusivo ao idoso, contudo, os indivíduos que mais se beneficiam desse dispositivo assistencial, além dos idosos, são os sujeitos acamados, com condiçôes crônicas de saúde descompensadas, complicaçôes das doenças crônicas e que requerem cuidados paliativos.

O Padi do município do Rio de janeiro está em franca expansão desde o ano de $2010^{14}$ e atualmente conta com cinco bases alocadas estrategicamente nos cinco principais hospitais municipais das áreas programáticas do município, estando integrado, além dos hospitais-sede, aos serviços da rede, tais como clínicas de saúde da família, equipes do núcleo de apoio à estratégia de saúde da família, centros municipais de saúde, policlínicas, unidades de pronto atendimento (UPA) e coordenaçóes de emergência regionais.

O eixo principal do Padi é evitar a hospitalização desnecessária, com a otimização dos leitos hospitalares e a racionalização dos recursos financeiros, tendo a finalidade de dar continuidade ao cuidado nos domicílios dos indivíduos previamente internados nos hospitais municipais e federais. O outro eixo é o suporte e 
matriciamento às açóes dos serviços de atenção primária do município, no qual as equipes desses serviços solicitam as vagas pelo sistema de regulação (Sisreg).

Para ser incluído no programa, o usuário necessita cumprir alguns critérios que garantem a operacionalidade e viabilidade das açóes do Padi e a segurança do paciente dentro do serviço. A partir da inclusão, o cuidado passa a ser compartilhado entre os pilares: unidade primária de saúde, equipes de atenção domiciliar, rede de apoio de exames diagnósticos e, quando necessário, unidades de urgência e emergência e/ou hospital geral.

Figura 1: Critérios elegibilidade no PAD do Rio de Janeiro.

\begin{tabular}{|c|c|}
\hline Critérios de Inclusão no PADI & Inclusão no PADI \\
\hline$\longrightarrow$ & Ser acamado; \\
\hline$\longrightarrow$ & Ser residente no município do Rio de Janeiro; \\
\hline$\rightarrow$ & $\begin{array}{l}\text { Ser oriundo das Unidades de Saúde que } \\
\text { compõem o SUS; }\end{array}$ \\
\hline & $\begin{array}{l}\text { Possuir um responsável que assine o Termo } \\
\text { de Compromisso do PADI; }\end{array}$ \\
\hline & $\begin{array}{l}\text { Possuir um responsável que assuma a função } \\
\text { de cuidador seja familiar ou não; }\end{array}$ \\
\hline$\longrightarrow$ & $\begin{array}{l}\text { Residir em domicílio com recursos mínimos } \\
\text { de infraestrutura; }\end{array}$ \\
\hline & $\begin{array}{l}\text { Ter encaminhamento médico para o PADI, } \\
\text { garantindo a estabilidade clínica; }\end{array}$ \\
\hline
\end{tabular}

Fonte: Elaboração dos autores.

Assim como preconizado pelas publicações do Melhor em Casa ${ }^{5,6,8}$, a atuação no Padi é interdisciplinar, oferecida por uma equipe composta por profissionais de várias formaçóes, o que proporciona aos usuários um suporte integral às suas demandas e complementação da assistência que é oferecida pelos demais pontos de atenção da rede de saúde.

As equipes são divididas em Emad e Emap, como recomenda a Portaria 963/2013 do Ministério da Saúde . Atualmente há 11 Emad e 5 Emap vinculadas ao Padi, distribuídas de forma equânime no município. As equipes realizam procedimentos técnicos intradomiciliares e, prioritariamente, capacitam o cuidador, de maneira que este assuma a corresponsabilidade e contribua com a realização das condutas/orientaçóes propostas pelos profissionais, atendendo, assim, às demandas do paciente e melhorando a qualidade de vida e celeridade no processo de recuperação.
A educação permanente através da capacitação dos cuidadores é um aspecto importante dentro da atenção domiciliar, uma vez que a maioria é composta por familiares sem formação na área da saúde. Mensalmente, oficinas de capacitação são realizadas, fornecendo treinamento e orientaçóes aos cuidadores. As dúvidas são sanadas com a participação de todos os profissionais, servindo também espaço propício à aproximação entre os atores e ao apoio para o cuidado.

A tarefa de ensinar os familiares a cuidar do paciente é essencial para que o cuidado domiciliar se concreti$\mathrm{ze}^{11} \mathrm{e}$, dessa maneira, o cuidado compartilhado no Padi se torne uma estratégia para envolver os cuidadores no tratamento, proporcionando autonomia e reforço do empoderamento desses sujeitos. Isso também é um diferencial da atenção domiciliar, já que o paciente e sua família participam ativamente no processo de cuidados ${ }^{7}$.

A assistência oferecida no Padi é temporária. No período de coleta de dados deste estudo, o tempo médio de permanência era de 120 dias. Nas publicaçôes referentes ao programa Melhor em Casa ${ }^{5,6,8}$, o Ministério da Saúde não estipula o tempo médio ideal de permanência no SAD. Ao mesmo tempo, esse aspecto é previsto como indicador sugerido para monitorar a resolutividade e a articulaçáo do serviço com a rede de atenção à saúde ${ }^{5,8}$.

A alta do serviço ocorre por reabilitação clínica/funcional, adaptação do cliente à condição de saúde e do cuidador às intervençóes propostas, apenas sob o parecer de todos os profissionais das equipes. Em alguns casos, quando a necessidade de cuidado domiciliar ainda existe, esse tempo de permanência é superado. Quando ocorre a alta no Padi, o indivíduo é referenciado para acompanhar a unidade de atenção primária referência, que geralmente é uma Clínica da Família.

\section{A atuação do enfermeiro no Padi}

Dentro do Padi no Rio de Janeiro, o enfermeiro tem participaçáo nos cargos assistenciais e de gestão. Dentro da assistência, que é objeto da observação sistemática deste estudo, esse profissional desenvolve açóes no âmbito da promoção, prevenção, diagnóstico, tratamento e reabilitação de saúde, tendo como ênfase as açóes direcionadas ao paciente, à família e ao ambiente (domicílio).

A atuação assistencial do enfermeiro no PAD tem congruência com as atividades desempenhadas na atenção primária a saúde, diferenciando-se pelo fato de ser um cuidado mais intensivo e contínuo. Ao mesmo tempo que oferece maior autonomia ao enfermeiro, o cuidado no domicílio exige novas competências, habilidades e uso de novas tecnologias assistenciais ${ }^{10}$, tendo 
como princípios básicos o cuidar, o ajudar a cuidar, o orientar e o encaminhar ${ }^{15}$.

No Padi o enfermeiro age na avaliação de saúde, além de prescrever, implementar e julgar condutas clínicas através da sistematizaçáo da assistência de enfermagem. Inicialmente, o paciente e as condiçóes da família são alvos de avaliaçóes multidimensionais, em que os problemas e as necessidades são identificadas e priorizados para que as prescriçóes de cuidados sejam planejadas, propostas e aplicadas de maneira interdisciplinar.

A primeira consulta no Padi é realizada pelo enfermeiro, juntamente com o técnico de enfermagem da equipe, tendo o intuito principal de avaliar o atendimento aos critérios de inclusão para açóes de atenção domiciliar e o estado do perfil clínico-funcional do paciente. Dessa maneira, a demanda de cuidado do paciente é classificada em um dos seguintes níveis de atenção domiciliar: $\mathrm{AD} 1, \mathrm{AD} 2$ ou $\mathrm{AD} 3$, e o paciente recebe uma pontuação a partir do escore de Barthel, que também é aplicado pelo enfermeiro.

$\mathrm{Na}$ modalidade AD1, o cuidado é oferecido pela atenção primária à saúde; nas modalidades de AD2 e AD3, o cuidado é implementado pelas Emad e Emap do Padi, de acordo com o território e área geográfica do município do Rio de Janeiro, estando o enfermeiro presente nessas três modalidades de atenção. Ressaltase que a assistência oferecida pelas equipes do Padi não excluem o usuário da cobertura de atenção primária à saúde, sendo a atenção domiciliar apartada em modalidades com o intento de se enfatizar a existência de diferentes complexidades assistenciais ${ }^{6}$.

A modalidade AD1 é destinada a usuários com condiçóes de saúde estabilizadas, com dificuldade de locomoção até o serviço de saúde e com demanda de cuidado menos complexa, que seja abordável pelas unidades primárias. A AD2 é destinada a indivíduos que, além de não poderem comparecer ao serviço de saúde, apresentam demandas complexas de assistência, tais como o manejo de feridas complexas, abscessos, sondas, ostomias, entre outros. A AD3 é indicada para sujeitos que, além de apresentarem as situaçóes anteriores, ainda demandam suporte ventilatório não invasivo, métodos dialíticos e/ou paracentese ${ }^{6}$.

Dessa maneira, a partir da classificação do perfil clínico-funcional do paciente e a elegibilidade para o cuidado pelas equipes de atençáo domiciliar do Padi (Emad e Emap), as demandas biopsicossociais do paciente e família observadas pelo enfermeiro são discutidas em equipe, através das reunióes semanais que acontecem no serviço. Os profissionais dimensionam, entáo, a frequência de visitas domiciliares de acordo com as necessidades do paciente e família.

As atividades principais do enfermeiro nas visitas são concernentes ao paciente, à família e ao ambiente (domicílio). Foi observado que as atividades relacionadas diretamente ao paciente incluíram a avaliação do seu estado geral, com a apreciação dos sinais vitais atuais, anteriores e dos registrados pela família. O enfermeiro também orientou e administrou medicamentos, com revisão do prontuário e prescrição domiciliar, além da prevenção aos eventos iatrogênicos associados à polifarmácia e implementação dos cuidados paliativos.

Concernente, ainda, sobre a abordagem do enfermeiro ao usuário dentro do Padi, este também solicitou exames laboratoriais e de imagem diversos, demonstrando, assim, resolutividade e autonomia profissional. O cuidado com feridas, em especial as úlceras por pressão, além de sondas e cateteres, também se mostrou um foco da abordagem desse profissional dentro do Padi, frente ao risco de agravamento clínico-funcional que essas situações promovem.

O enfermeiro também participa das discussões sobre viabilidade e adesão das condutas traçadas pela equipe interdisciplinar, sendo um dos principais diferenciais da atenção domiciliar, uma vez que há intervenções baseadas em um olhar multidimensional e interdisciplinar. Essa integração das equipes da atençáo domiciliar propiciam um melhor desempenho profissional e maior satisfação dos pacientes e da família ${ }^{10}$.

Quanto à assistência à família, observou-se que a atuaçáo do enfermeiro incluiu principalmente as açóes de educação em saúde, a análise da dinâmica familiar, a identificação precoce, a mediaçẫo das situaçôes familiares de conflito e a prevenção e abordagem das situaçóes de violência ao paciente. A ênfase está relacionada à educação em saúde, que é abordada a cada visita do enfermeiro ao domicílio, uma vez que é necessário capacitar o cuidador e o paciente.

Os familiares e cuidadores dentro do Padi recebem capacitações mensais em grupo, no intuito de trabalhar as dificuldades singulares a partir do compartilhamento de experiências de um coletivo. As oficinas de capacitação também funcionam como espaço de apoio para as demandas psíquicas e sociais do cuidador. A capacitação do cuidador, que é organizada, em maioria, pelo enfermeiro, se mostra importante para oferecer subsídios para o cuidado domiciliar, além de possibilitar a mudança no comportamento e estilo de vida do paciente e do cuidador ${ }^{16}$.

As atividades relacionadas ao ambiente de cuidado se referem ao descarte correto de resíduos da assistência e orientaçóes quanto a adaptaçóes possíveis no domicílio, com a finalidade de proporcionar um ambiente salubre e propício à reabilitação, uma vez que o domicílio pode não ser adequado para o cuidado, exigindo assim adaptações daqueles que irão realizá-lo ${ }^{11}$.

Outro aspecto do cuidado de enfermeiro é o dimensionamento de pessoal da enfermagem, atividade privativa do enfermeiro, em que são dimensionados enfermeiros e técnicos de enfermagem de acordo com a demanda dos usuários e de acordo com a quantidade de atividades prescritas 
por ele. Essas açóes a serem realizadas e orientadas pela equipe técnica de enfermagem foram principalmente a realizaçáo de enteroclismas, coleta de materiais para exames, orientaçôes sobre higiene e prevenção de feridas.

Diante de um relevante e emergente cenário de práticas, evidencia-se que o enfermeiro possui participação ativa na prestação da atenção domiciliar, contribuindo para as açôes desde a inclusão do usuário até a alta do serviço. Ressalta-se que assim como recomendado pelas publicaçôes da atenção domiciliar ${ }^{5,6,8}$, o trabalho desse profissional deve estar adicionado ao trabalho de outros profissionais, dentro de uma equipe multiprofissional e interdisciplinar para que as açôes sejam multidimensionais, integrais e resolutivas.

\section{Conclusão}

A atenção domiciliar está emergindo como uma relevante modalidade assistencial à saúde e um campo de práticas para o enfermeiro. Desse modo, compreender as principais atribuiçóes desse profissional nesse serviço contribui para uma atuação pautada na perícia, bem como nas delimitaçóes éticas e legais de cada profissão, além de garantir subsídios à formação e aperfeiçoamento profissional para essa área.

Este estudo contribuiu para explorar e delimitar as principais atribuiçóes desempenhadas pelo profissional enfermeiro dentro de um serviço de atenção domiciliar do SUS direcionado a idosos. As principais limitaçóes foram a realização em um único programa e a abordagem a somente uma classe profissional. Recomenda-se, portanto, estudos multicêntricos posteriores que ampliem o conhecimento sobre essa modalidade de atençáo à saúde e que abordem as principais atribuiçôes dos profissionais das equipes multiprofissionais desse serviço.

\section{Referências}

1. Moraes EN. Atenção à saúde do idoso: aspectos conceituais. Brasília: OPAS; 2012.

2. Instituto Brasileiro de Geografia e Estatística. Censo Demográfico 2010. Rio de Janeiro: IBGE; 2012.

3. Day CB, Paskulin LMG. Benefícios da atenção domiciliar ao idoso portador de dano crônico. Rev Enferm UERJ. 2013;21(3):384-90.

4. Geib LTC. Determinantes sociais da saúde do idoso social. Ciênc Saúde Coletiva. 2012;17(1):123-33.
5. Brasil. Ministério da Saúde. Manual de monitoramento e avaliação: Programa Melhor em Casa. Brasília: Ministério da Saúde; 2014.

6. Brasil. Conselho Federal de Fisioterapia e Terapia. Portaria no 963/GM, de 27 de maio de 2013. Redefine a Atenção Domiciliar no âmbito do Sistema Único de Saúde. Diário Oficial [da] República Federativa do Brasil. Brasília: Ministério da Saúde, 2013 [citado em 2016 nov 9]. Disponível em: http://coffito.gov.br/nsite/?p=3348.

7. Brito MJM, Andrade AM, Caçador BS, Freitas LFC, Penna CMM. Atenção domiciliar na estruturação da rede de atenção à saúde: trilhando os caminhos da integralidade. Esc Anna Nery. 2013;17(4):603-10.

8. Brasil. Ministério da Saúde. Caderno de atenção domiciliar. Brasília: Ministério da Saúde; 2012.

9. Andrade AM, Brito MJM, Silva KL, Montenegro LC, Caçador BS, Freitas LFC. Organização das redes de atenção à saúde na perspectiva de profissionais da atenção domiciliar. Rev Gaúcha de Enferm. 2013;34(2):111-7.

10. Alencar LH, Martins AAA, Noronha FAT, Matias GFS, Bezerra IMP, Antão JYFL, et al. Assistência de enfermagem frente aos cuidados domiciliares: a percepção dos profissionais. Trabalho apresentado no II Congresso - Gestão, Educação e Promoção da Saúde: CONVIBRA; 24 a 26 de outubro de 2013 [citado em 2016 nov 9]; São Paulo, SP, Brasil. Disponível em: http://www.convibra.com.br/ upload/paper/2013/71/2013_71_7580.pdf.

11. Hermann AP, Nascimento JD, Lacerda MR. Especificidades do cuidado domiciliar apreendidas no processo de formação profissional do enfermeiro. REME Rev Min Enferm. 2014;18(3):545-50.

12. Bôas MLCV, Shimizu HE. Tempo gasto por equipe multiprofissional em assistência domiciliar: subsídio para dimensionar pessoal. Acta Paul Enferm. 2015;28(1):32-40.

13. Silva KL, Sena RR, Silva PM, Souza CG, Martins ACS. Atuação do enfermeiro nos serviços de atenção domiciliar: implicaçóes para o processo de formação. Ciênc Cuidado e Saúde. 2014;13(3):503-10.

14. Rio de Janeiro. Portal da Prefeitura da Cidade do Rio de Janeiro. Secretaria Municipal de Saúde - SMS. Programa de Atenção Domiciliar ao Idoso - PADI. Rio de Janeiro; 2013 [citado em 2016 jan 16]. Disponível em: http://www.rio. rj.gov.br/web/sms/padi.

15. Machado ALG, Silva MJ, Freitas CHA. Assistência domiciliária em saúde: um olhar crítico sobre a produção científica de enfermagem. Rev Bras Enferm. 2011;64(2):365-9.

16. Valle ARMC, Andrade D. Habilidades e atitudes do enfermeiro na atenção domiciliar: bases para a prevenção dos riscos de infecção. REME Rev Min Enferm. 2015;19(2):67-78.

\section{Como citar este artigo:}

Ribeiro DFS, Abreu GP. Atribuiçôes do enfermeiro em um programa de atenção domiciliar do sistema único de saúde. Rev. Aten. Saúde. 2017;15(52):55-60. 